\title{
The mechanism of the colour shift of astaxanthin in alpha- crustacyanin as investigated by C-13 MAS NMR and specific isotope enrichment
}

Weesie, R.J.; Verel, R.; Jansen, F.J.H.M.; Britton, G.; Lugtenburg, J.; Groot, H.J.M. de

\section{Citation}

Weesie, R. J., Verel, R., Jansen, F. J. H. M., Britton, G., Lugtenburg, J., \& Groot, H. J. M. de. (1997). The mechanism of the colour shift of astaxanthin in alphacrustacyanin as investigated by C-13 MAS NMR and specific isotope enrichment. Pure And Applied Chemistry, 69(10), 2085-2090. doi:10.1351/pac199769102085

Version:

Publisher's Version

License:

Licensed under Article 25fa Copyright Act/Law (Amendment Taverne)

Downloaded from: $\quad$ https://hdl.handle.net/1887/3238825

Note: To cite this publication please use the final published version (if applicable). 


\title{
The mechanism of the colour shift of astaxanthin in $\alpha$-crustacyanin as investigated by ${ }^{13} \mathrm{C}$ MAS NMR and specific isotope enrichment
}

\author{
Roland J. Weesie, ${ }^{\mathrm{a}, \mathrm{b}}$ Rene Verel, ${ }^{\mathrm{a}}$ Frans Jos H.M. Jansen, ${ }^{\mathrm{a}}$ George Britton, ${ }^{\mathrm{b}}$ Johan Lugtenburg, ${ }^{\mathrm{a}}$ \\ and Huub J.M. de Groot ${ }^{\mathrm{a}}$ \\ ${ }^{a}$ Leiden Institute of Chemistry, Gorlaeus Laboratories, P.O. Box 9502, 2300 RA Leiden, NL \\ ${ }^{b}$ Department of Biochemistry, School of Biological Sciences, University of Liverpool, Crown \\ Street, Liverpool L69 3BX, UK
}

\begin{abstract}
By selective isotope enrichment of astaxanthin, MAS NMR and semi-empirical modelling, ligand-protein interactions associated with the red shift in $\alpha$-crustacyanin, the major blue astaxanthin binding carotenoprotein complex from the carapace of the lobster Homarus gammarus, have been analysed. ${ }^{13} \mathrm{C}$ Magic Angle Spinning (MAS) NMR spectra were obtained after reconstitution with astaxanthins labelled in the centre of the molecule or at the two keto groups. The MAS data reveal electrostatic polarizations of the conjugated chain. In addition, solid state NMR results for pure unlabelled astaxanthin can be compared with natural abundance ${ }^{13} \mathrm{C}$ MAS data for canthaxanthin and $\beta$-carotene, to address the effect of the ring functionalities on the electronic properties of the polyene chain. Quantum chemical calculations were performed to reconcile the MAS data with one of several simple and straightforward mechanisms for the colour shift. The results point towards a colour shift mechanism in which the astaxanthin may be doubly charged, possibly by a double protonation of the two ring keto groups.
\end{abstract}

\section{INTRODUCTION}

$\alpha$-Crustacyanin is the major blue protein complex in the carapace of the lobster Homarus gammarus. It may be considered to represent an entire class of similar complexes in crustaceans and other organisms that may serve as a basic defence system for these animals, since it provides them with a colour adapted to their natural living environment, the bottom of coastal seawaters. In $\alpha$-crustacyanin the colour is determined by the carotenoid astaxanthin (Fig. 1), which is subject to specific interactions with the protein, in order to increase the wavelength of maximum optical absorption from $\lambda_{\max } \sim 480 \mathrm{~nm}$, depending on the solvent, to $\lambda_{\max }=632 \mathrm{~nm}$ in the protein complex. This red shift is among the largest known in Nature.

Recently, we have started a systematic joint investigation aimed at elucidating the precise molecular mechanisms behind the bathochromic shift and the associated ligand-protein interactions of the astaxanthin in the $\alpha$-crustacyanin, all of which are essentially unknown at present. In these in-depth investigations into the chromophore-protein interactions at the atomic level, extensive use is made of the relatively new Magic Angle Spinning (MAS) NMR and specific isotope labelling approach. This is the method of choice to obtain NMR access to large protein complexes like the $\alpha$-crustacyanin, which is beyond the scope of standard solution NMR technology because of its large size, around $\sim 320 \mathrm{kDa}$ (ref. 1).

Over the past two decades, MAS NMR in conjunction with site-specific isotope enrichment has been helpful in elucidating molecular mechanisms of colour shifts of retinylidene chromophores in rhodopsins, chemical energy conversion proteins that are involved in, for instance, visual signal transduction and lightdriven ion pumping (ref. 2). Because of the obvious structural and electronic analogies between 


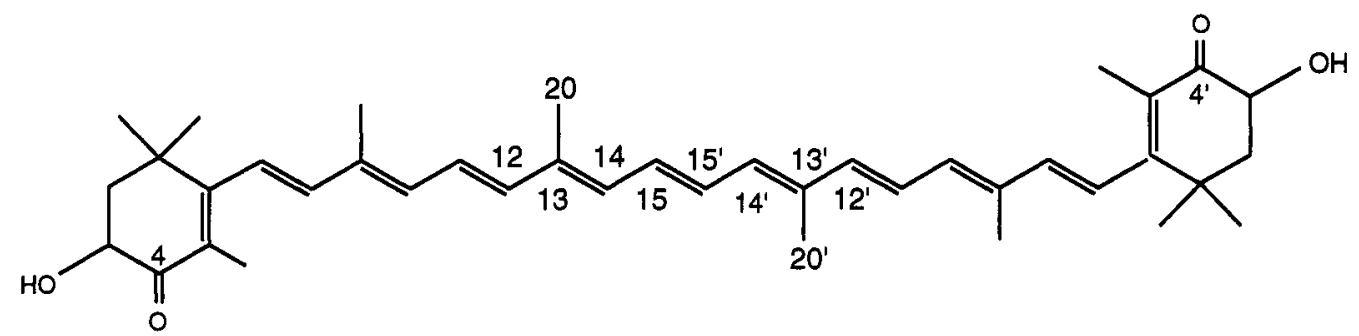

Fig. 1 Structure of astaxanthin. The positions of the ${ }^{13} \mathrm{C}$ labels used for the MAS work are indicated with numbers

carotenoids and retinals, it can, in principle, be anticipated that mechanisms involved in tuning the retinal properties can be considered candidates for the tuning of the $\lambda_{\max }$ of carotenoids as well. Conversely, it is of interest to know if the mechanisms that have been demonstrated to operate in rhodopsins are more generally found. This may point towards the existence of generic types of ligand-protein interactions associated with the special electronic and structural motif of an electrostatically polarized conjugated chain in a protein or complex. Such information is important. For instance, it may help towards understanding drug-receptor binding processes mediated by pharmacophores.

Crustacyanins are thus good models for studying specific types of ligand-protein interactions that play such fundamental roles in many biological and biochemical processes, provided that the molecular mechanisms behind the large bathochromic shift and the nature of the corresponding protein-chromophore interactions can be resolved. Whilst the formulation of working hypotheses based on earlier investigations of similar biological phenomena in other proteins can be useful and may prove effective, it is important to take into account what is known or has been proposed regarding the structural basis of the colour shift in crustacyanins at the molecular and atomic level. Reconstitution studies have revealed that the central methyl groups and both ring keto functionalities of the astaxanthin are crucial for its binding in $\alpha$ crustacyanin and for the full red shift (ref. 3), while resonance Raman studies on the intact $\alpha$-crustacyanin have provided compelling evidence that the astaxanthin behaves spectroscopically as a monomer (ref. 4), despite the fact that a complete hexadecameric complex, which binds 16 carotenoid molecules, is required to obtain the deep blue colour! Finally, a structural model has been presented in the literature for the $\beta$ crustacyanin dimeric subunit, which has the two astaxanthins each capped on one end by an apolar protein environment while the other end is exposed to the medium (ref. 5). This model suggests a pronounced asymmetry in the binding, particularly with respect to the ring keto functionalities.

Building upon this limited information, we have started to place ${ }^{13} \mathrm{C}$ NMR labels in the central part of the chromophore and at the keto carbons by total synthesis (ref. 6). The positions of the labels are indicated in Fig. 1. In parallel, quantum chemical calculations have been performed to investigate what type of interaction mechanisms may have to be invoked for generation of the large colour shift in a single astaxanthin molecule and to model the NMR chemical shifts observed in the MAS experiments. Finally, to provide an appropriate background for the NMR and quantum chemical modelling studies on the protein, the MAS signals of pure solid astaxanthin are analysed at the level of the principal components of the chemical shift anisotropies and are compared with data collected for canthaxanthin, which lacks the 3-hydroxy groups, and $\beta$-carotene, which has no oxygen functionalities. The purpose of the present contribution is to provide a brief summarizing report on these studies, which are now well underway and will be published in detail elsewhere. This paper complements the more general survey presented in the preceding review paper (ref. 3).

\section{MAS NMR OF ASTAXANTHIN, CANTHAXANTHIN AND $\beta$-CAROTENE}

A ${ }^{13}$ C MAS NMR signal of a polycrystalline sample consists of a centreband at the isotropic shift $\sigma_{1}$, flanked by sidebands at integral multiples of the spinning speed $n \omega_{\mathrm{r}} / 2 \pi$. The intensities of the sidebands, relative to the centreband, are due to the anisotropy of the chemical shift, which in turn is related to the local electronic structure around the ${ }^{13} \mathrm{C}$ nuclei responsible for the NMR signal (ref. 7). When the spinning is slow, $\omega_{\mathrm{r}} / 2 \pi<\delta$ (the anisotropy parameter), sidebands adjacent to the centreband appear very strong in the spectrum. For fast spinning, $\omega_{\mathrm{r}} / 2 \pi>\delta$, most of the NMR signal intensity is in the centreband with 


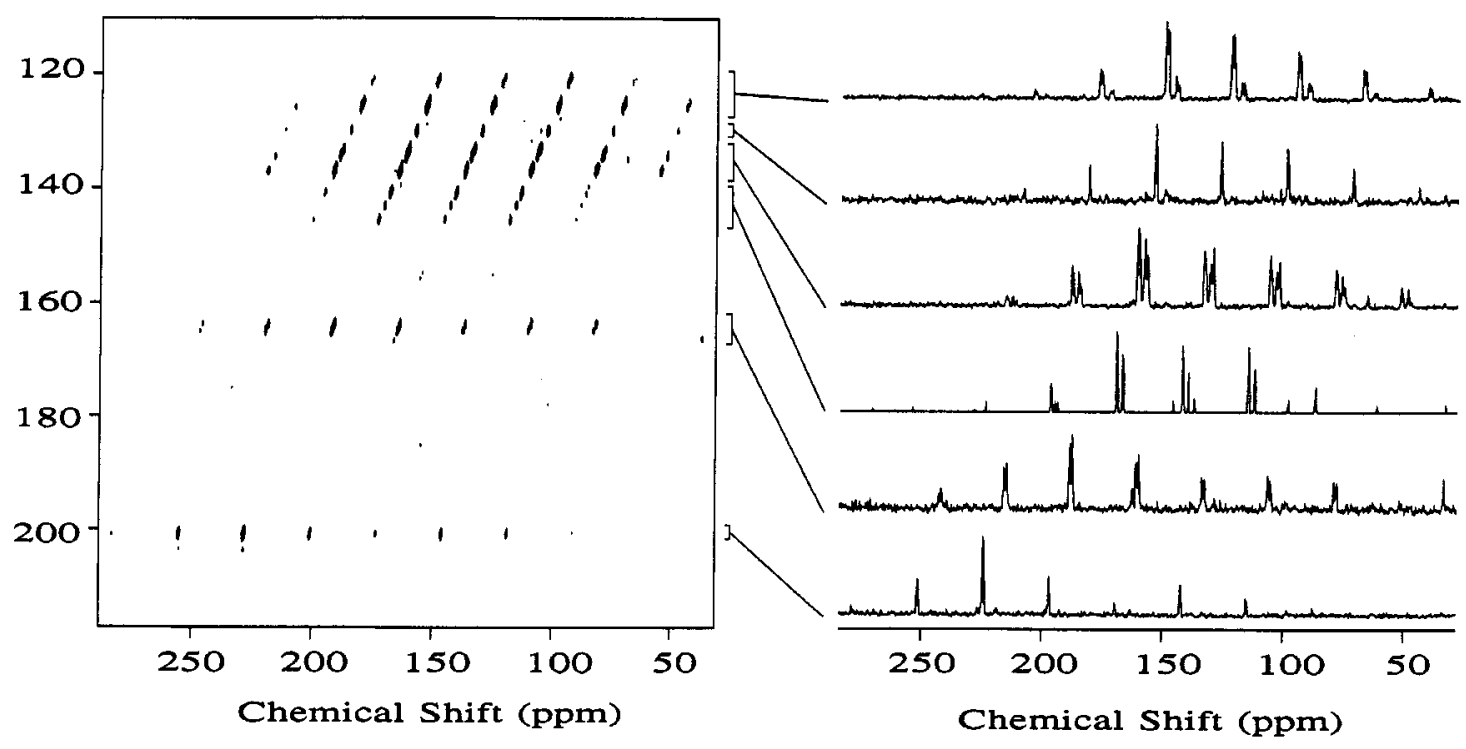

Fig. 2 TOSS - reversed TOSS spectrum of all-E-astaxanthin, collected with $\omega_{\mathrm{r}} / 2 \pi=2750 \mathrm{~Hz}$. To the right are shown projections of regions of the 2-D dataset.

shift $\sigma_{\mathrm{i}}$. Alternatively, TOtal Sideband Suppression (TOSS) pulse sequences can be applied to eliminate the sidebands even at low spinning speeds (ref. 8). This is most conveniently demonstrated in Fig. 2, which shows the olefinic and carbonyl regions of a 2-D TOSS - reversed TOSS spectrum of pure unlabeled R,R'-all-E-astaxanthin, collected with a $\omega_{\mathrm{r}} / 2 \pi=2750 \mathrm{~Hz}$. In the vertical dimension, TOSS sequences were applied to suppress the sidebands, while in the horizontal dimension sideband patterns are detected, characteristic of the low-speed MAS experiment (refs. 9-10). For the pure carotenoid the natural abundance of $\sim 1 \%{ }^{13} \mathrm{C}$ is sufficient to provide good signal-to-noise for this type of 2-D experiment.

To the right of the 2-D contour plot in Fig. 2, projections of regions of the 2-D spectrum are shown. Due to the high resolution in the vertical dimension, the overlap between signals from the carbons in the conjugated backbone is significantly reduced. The projections can be deconvoluted by using theoretical MAS patterns to yield the $\sigma_{\mathrm{i}}$ and a characterization of the anisotropy, through the principal components of the shift tensor, $\sigma_{11}, \sigma_{22}$ and $\sigma_{33}$ (refs. 11-12). Such analyses have been performed for unlabelled samples of astaxanthin, canthaxanthin and $\beta$-carotene, to address the general differences in electronic properties of these molecules, and to provide an experimental basis for a comparison with the semiempirical calculations.

At the level of the isotropic shift, pronounced differences occur between, on the one hand, conjugated carbons adjacent to the keto functionalities in astaxanthin and canthaxanthin and, on the other hand, their counterparts in $\beta$-carotene. This is due to the electrostatic polarization of the conjugated chain by the strongly electronegative keto groups. In this respect, the solid state results reproduce very well the results from solution NMR studies on these molecules (ref. 13). The positive charge is stored mainly on the 4,4' keto carbons and the $6,6^{\prime}$ carbons, two bonds further into the conjugated chain. These nuclei are subject to downfield shifts of $\sim 70 \mathrm{ppm}\left(4,4^{\prime}\right)$ and $25 \mathrm{ppm}\left(6,6^{\prime}\right)$ due to the charge effect.

The charge delocalization also affects the principal components of the chemical shift tensors measured for these carbons. The analysis of the lowest trace in the right part of Fig. 2, representing the partial projection of the spectral region around $200 \mathrm{ppm}$, which comprises the signal of the $4,4,{ }^{13} \mathrm{C}$, yields $\sigma_{11}=$ $85 \mathrm{ppm}, \sigma_{22}=240 \mathrm{ppm}$ and $\sigma_{33}=270 \mathrm{ppm}$. These values are characteristic for a keto carbon and the shielding tensor is nearly axially symmetric since $\sigma_{22} \sim \sigma_{33}$ (ref. 14). From the partial projection around $165 \mathrm{ppm}$, representing the signal from the the $6,6,{ }^{13} \mathrm{C}$, it can be deduced that both $\sigma_{22}$ and $\sigma_{33}$ are shifted downfield by $\sim 40 \mathrm{ppm}$ relative to their values in $\beta$-carotene, $\sigma_{22}=140 \mathrm{ppm}$ and $\sigma_{33}=225 \mathrm{ppm}$, while $\sigma_{11} \sim 50 \mathrm{ppm}$ in all three compounds. Perhaps surprisingly, the effect of the $3-\mathrm{OH}$ on the conjugated chain in astaxanthin when compared to canthaxanthin is almost negligible. Interestingly, the $5,5^{\circ}-{ }^{13} \mathrm{C}$ is 


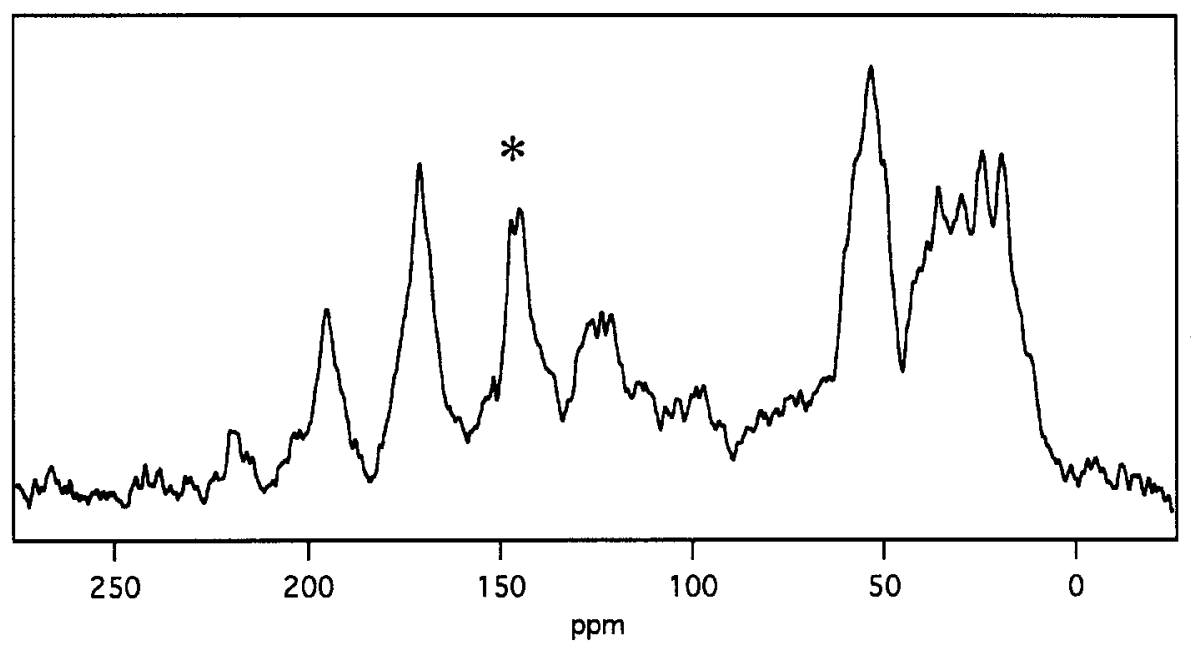

Fig. $3 \mathrm{CP} / \mathrm{MAS}$ spectrum of $\alpha$-crustacyanin reconstituted with $\left[12,12^{\prime}-{ }^{13} \mathrm{C}_{2}\right]$ astaxanthin, recorded at $\omega_{\mathrm{r}} / 2 \pi=6.00$ $\mathrm{kHz}$, at a sample temperature $\mathrm{T} \sim 230 \mathrm{~K}$.

only marginally shifted and yields $\sigma_{22}=140 \mathrm{ppm}$ in all three compounds, which provides strong evidence that the ring conformations in solid astaxanthin are 6-s-cis, like in $\beta$-carotene and canthaxanthin.

\section{${ }^{13}$ C MAS NMR ANALYSIS OF LABELLED ASTAXANTHIN IN $\alpha$-CRUSTACYANIN}

It has been shown in the past that CP/MAS NMR and selective isotope enrichment allow a systematic and routine investigation of ligand-protein interactions of essentially unperturbed and unmodified protein complexes at atomic resolution (ref. 15). To prepare samples for the MAS experiments, $\alpha$-crustacyanin was extracted from ground lobster carapace, purified, and reconstituted with synthetic astaxanthins containing ${ }^{13} \mathrm{C}$ labels (refs. 1, 16). The $\alpha$-crustacyanin hexadecamer is a soluble protein complex. For the solid-state NMR experiments, concentrated buffered solutions containing $\sim 20 \mathrm{mg}$ of the reconstituted $\alpha$ crustacyanin were transferred into $4 \mathrm{~mm} 100 \mu \mathrm{l}$ MAS rotors and frozen to $\sim 230 \mathrm{~K}$, which is considered an optimal temperature for these experiments, necessary to obtain rigid solid samples (ref. 1). Just before and immediately after the acquisition of the MAS data, optical spectra were taken of small aliquots of the NMR samples, which confirmed that the preparations remained in good condition.

As an example, the ${ }^{13} \mathrm{C}$ CP/MAS NMR spectrum of $\alpha$-crustacyanin reconstituted with $\left[12,12,-{ }^{13} \mathrm{C}_{2}\right]$ astaxanthin is shown in Fig. 3. This spectrum is considerably more complex than the projections shown in the right part of Fig. 2, for the pure unlabelled carotenoid. This is due to the natural abundance background from the protein, with its aliphatic resonances spread between 0 and $80 \mathrm{ppm}$, the aromatic and olefinic signals around $128 \mathrm{ppm}$, and finally a pronounced MAS pattern from the peptide carbonyls with $\sigma_{\mathrm{i}} \sim 172$ $\mathrm{ppm}$ and strong sidebands at 232 and $112 \mathrm{ppm}$. From such spectra, the contribution from the labels can be identified by subtracting data from an unlabelled $\alpha$-crustacyanin sample, collected with the same experimental parameters (ref. 1). In Fig. 3 the centreband response from the 12,12' labels is indicated with an asterisk.

${ }^{13} \mathrm{C}$ MAS NMR spectra of $\alpha$-crustacyanin were obtained after reconstitution with astaxanthin specifically labelled with ${ }^{13} \mathrm{C}$ at the positions $4,4^{\prime}, 12,12^{\prime}, 13,13^{\prime}, 14,14^{\prime}, 15,15^{\prime}$ or $20,20^{\prime}$ (cf. Fig. 1). Comparison of the ${ }^{13} \mathrm{C}$ chemical shifts of the labels when in the protein with these when in the pure solid reveals downfield shifts at the positions $12,12^{\prime}$ of 4.9 and $7.0 \mathrm{ppm}$. For the $13,13^{\prime}$ positions small upfield shifts of $1.9 \mathrm{ppm}$ were observed upon binding to the protein. The shift differences for these 12,12' positions are in line with the results for positions $14,14^{\prime}$, i.e. 3.9 and $6.8 \mathrm{ppm}$. Similar to positions $13,13^{\prime}$, the 15 and 15 ' carbon signals exhibit a very small protein-induced shift of $0.6 \mathrm{ppm}$. The keto functionalities and the central methyl groups are crucial binding motifs, but only small shifts, of 2.4 and $-0.4 \mathrm{ppm}$, respectively, were detected for the 4,4' and 20,20 ' positions. 
The chromophore is asymmetrically bound with the conjugated chain polarized by the protein. A change in charge density of 1 electronic equivalent corresponds to a downfield shift of $\sim 155 \mathrm{ppm}$ for olefinic carbons (ref. 17). The NMR shifts for the 12,12' and 14,14' positions translate into positive fractional charge densities of $0.03-0.05$. In contrast, a very small net increase of electron density is detected for the $13,13^{\prime}$ and $15,15^{\prime}$ positions. The data suggest that the charge effects on the two halves of the chromophore are somewhat different, but the sign and order of magnitude of the shifts is the same. Thus, according to the CP/MAS NMR, the main perturbation of the chromophore has a symmetric character, whilst an asymmetry may be associated with the smaller perturbation. This is of interest, in view of the molecular model that proposes a predominant structural asymmetry (ref. 5).

\section{SEMI-EMPIRICAL MODELLING OF LIGAND-PROTEIN INTERACTIONS}

The CP/MAS data reveal modest bisymmetrical electrostatic polarizations in the central part of the conjugated system, and quantum chemical modelling can help to interpret these results in terms of a possible mechanism for the colour shift. By using a combination of molecular mechanics and semiempirical methods, including configuration interactions, a $\lambda_{\max }=441 \mathrm{~nm}$ is calculated with the AM1 parametrization for astaxanthin in vacuo, close to the experimental $\lambda_{\max } \sim 480 \mathrm{~nm}$ in solution. The difference reflects the fact that interactions with a solvent were not included in the calculations.

It appears that considerably strong charge perturbations of the polyene chain are necessary to provoke a red-shift of the order of $5000 \mathrm{~cm}^{-1}$ in a single astaxanthin monomer, matching the bathochromic shift of the astaxanthin in $\alpha$-crustacyanin. The moderate shift differences detected in the centre of astaxanthin in the complex strongly argue against intense electrostatic protein-chromophore interactions affecting the central region. Therefore, if additional charge polarization is responsible for the colour shift in $\alpha$-crustacyanin, it is likely to originate from the other crucial binding motif, the keto functionalities. Such a charge mechanism would be in line with the NMR results for the pure carotenoids. For instance, the difference between the optical transitions of $\beta$-carotene and astaxanthin, associated with the different groups attached to the rings, yields considerable partial fractional charges of $\sim+0.4$ and $\sim+0.16$ at the $4,4^{\prime}$ and the 6,6' positions, respectively. The effect on the centre of the conjugated chain is $2-5 \mathrm{ppm}$ for the $12,12^{\prime}$ positions and 1-2 ppm for the 13,13',14,14' and 15,15' positions. Based on the results obtained by these first NMR analyses, we are led to consider a mechanism for the colour shift in which at least both keto groups are strongly interacting with the protein and the carotenoid is doubly charged. This could be achieved by a full protonation of both keto functionalities, since, according to the modelling calculations, the effect of protonation on the shift of the C-4,4' chemical shifts is small, in agreement with the MAS NMR experiments. In addition, double protonation yields a calculated $\lambda_{\max }=609 \mathrm{~nm}$, close to the experimental $\lambda_{\max }=632 \mathrm{~nm}$, while the signs and orders of magnitude of all shift differences are correctly reproduced.

\section{CONCLUDING REMARKS}

${ }^{13} \mathrm{C}$ MAS NMR of selectively enriched astaxanthins in $\alpha$-crustacyanin, MAS model studies of carotenoids and semi-empirical quantum chemical modelling can be reconciled with a simple and straightforward charge mechanism for the bathochromic shift. Interestingly, charging by protonation is also responsible for a substantial part of the colour shift with respect to free retinal in the retinylidene proteins, which all contain protonated Schiff bases (ref. 2). There the protonation is remarkably stable. For instance, in $b R$ the Schiff base remains protonated until the protein unfolds and denatures at $\mathrm{pH} \sim 12$, while a Schiff base in solution has a $\mathrm{pK}_{\mathrm{a}} \sim 8$. It is thought that the local proton chemical potential around the Schiff base is raised and tuned by a complex counterion, consisting of several protic amino acid side chains connected to the Schiff base nitrogen through a water molecule (ref. 18). There is at present no direct evidence that a similar mechanism operates in the crustacyanins but, during the course of the NMR experiments on $\alpha$-crustacyanin reconstituted with $\left[14,14^{\prime}-{ }^{13} \mathrm{C}_{2}\right]$-astaxanthin it was observed that dehydration of the protein complex yields a purple species with the same chemical shifts for the labels as was found for the pure astaxanthin. Upon rehydration, the NMR spectrum of the protein complex and the labels is fully restored, which is intriguing. 


\section{ACKNOWLEDGEMENTS}

This research was financed in part by SCIENCE contract SC1-CT92-0813 of the European Commission and by the Netherlands Foundation for Chemical Research (SON-NWO). R.W. acknowledges a short term fellowship from the European Molecular Biology Organization. We are grateful to $C$. Erkelens for support with the NMR experiments, and a sample of optically pure $\left(R, R^{\prime}-a l l-E\right)$-astaxanthin was kindly provided by Gist Brocades Research Laboratories, Delft, The Netherlands.

\section{REFERENCES}

1. R.J. Weesie, D. Askin, F.J.H.M. Jansen, H.J.M. De Groot, J. Lugtenburg and G. Britton FEBS Lett. 362, 34-38 (1995).

2. J. Hu, R.G. Griffin and J. Herzfeld Proc. Natl. Acad. Sci. USA 91, 8880-8884 (1994).

3. G. Britton, R.J. Weesie, D. Askin, I.D. Warburton, L. Gallardo-Guerrero, H.J.M. de Groot, F.J.H.M. Jansen, J. Lugtenburg, J.-P. Cornard and J.-C. Merlin Pure Appl. Chem. preceding paper

4. V.R. Salares, N.M. Young, P.R. Carey and H.J. Bernstein J. Raman Spectroscopy 6, 282-288 (1977).

5. J.N. Keen, I. Caceras, E.E. Eliopoulos, P.F. Zagalsky and J.B.C. Findlay Eur. J. Biochem. 197, 407-417 and 202, 31-40 (1991).

6. F.J.H.M. Jansen, M. Kwestro, D. Schmidt and J. Lugtenburg Recl. Trav. Chim. Pays-Bas 113, 552-562 (1994).

7. R.G. Griffin, W.P. Aue, R.A. Haberkorn, G.S. Harbison, J. Herzfeld, E.M. Menger, M.G. Munowitz, E.T. Olejniczak, D.P. Raleigh, J.E. Roberts, D.J. Ruben, A. Schmidt, S.O. Smith and S. Vega in Physics of NMR Spectroscopy in Biology and Medicine, Proceedings of the Enrico Fermi International School of Physics, July 8-18, 1986 Varennea, Italy, North-Holland Press, Amsterdam (1988).

8. W.T. Dixon J. Chem. Phys. 77, 1800-1809 (1982).

9. A.C. Kolbert and R.G. Griffin Chem. Phys. Lett. 166, 87-91 (1990).

10. H. Geen and G. Bodenhausen J. Chem. Phys. 97, 2928-2937 (1992).

11. J. Herzfeld and A.E. Berger J. Chem. Phys. 73, 6021-6030 (1980).

12. H.J.M. De Groot, S.O. Smith, A.C. Kolbert, J.M.L. Courtin, C. Winkel, J. Lugtenburg, J. Herzfeld and R.G. Griffin $J$. Mag. Res. 91, 30-38 (1991).

13. G. Englert Helv. Chim. Acta 58, 2367- 2390 (1975).

14. K.W. Zilm and J.C. Duchamp in Nuclear Magnetic Shieldings and Molecular Structure (Tossell, J.A., ed.), pp. 315334. Kluwer Academic Publishers, Dordrecht, Boston, London (1993).

15. See e.g. H.J.M. De Groot in Advances in photosynthesis, Biophysical techniques in photosynthesis (J. Amesz and A.J. Hoff, eds.), Kluwer academic publishers, Dordrecht, Boston, London, 299-313 (1996).

16. P.F. Zagalsky Meth. Enzymol. 111B, 216-247 (1985).

17. H. Spiesecke and W.G. Schneider Tetrahedron Letters, 468-472 (1968).

18. H.J.M. De Groot, S.O. Smith, J. Courtin, E. Van den Berg, C. Winkel, J. Lugtenburg, R.G. Griffin and J. Herzfeld Biochemistry 29, 6873-6883 (1990). 